\title{
A High Resolution Chromosome Image Processor for Study Purposes, NIRS-1000:CHROMO STUDY, and Algorithm Developing to Classify Radiation Induced Aberrations
}

\author{
MIKIO YAMAMOTO ${ }^{1}$, ISAMU HAYATA ${ }^{2}$ AND SHINICHI FURUTA ${ }^{3}$ \\ ${ }^{1}$ Division of Physics, and ${ }^{2}$ Division of Radiation Hazards \\ National Institute of Radiological Sciences (NIRS), Anagawa-4, Inage-ku, \\ Chiba-shi 263 Japan ${ }^{3}$ Research Laboratory, Nikon Corporation \\ Nishi-ohi-1, Shinagawa-ku, Tokyo 140 Japan
}

\begin{abstract}
Automated cyto-genetics/Chromosome analysis/Radiation induced chromosome aberration/High definition video camera/Video microscope

Since 1989 we have promoted a project to develop an automated scoring system of radiation induced chromosome aberrations. As a first step, a high resolution image processing system for study purposes, NIRS-1000:CHROMO STUDY, has been developed. It is composed of:(1) CHROMO MARKER whose main purpose is to mark on images to make image data base, (2) CHROMO ALGO whose purpose is algorithm development, and (3) METAPHASE RANKER whose purposes are metaphase finding and ranking with a high power objective lens. However, METAPHASE RANKER is presently under development. The system utilizes a high definition video system so as to realize the best spatial resolution that is achievable with an optical microscope using an objective lens $(\times 100$, numerical aperture 1.4$)$. The video camera has 1024 effective scan lines to realize $0.1 \mu \mathrm{m}$ sampling on a specimen. The system resolution achieved on the hard copy is less than $0.3 \mu \mathrm{m}$ on a specimen. A preliminary algorithm has been developed to classify the aberrations on the system using projection information of gray level. The preliminary test results on excellent 10 metaphases show that the correct classification ratio is $92.7 \%$, that the detection rate of the aberrations is $83.3 \%$ and that the false positive rate is $6.1 \%$.
\end{abstract}

\section{INTRODUCTION}

Scoring of chromosome aberrations, dicentric and ring chromosomes, has the possibility to be a good biological radiation dosimeter. However, scoring is a work that consumes much time, especially at low radiation dose, because a great number of cells must be analyzed and almost all of these are normal cells. Therefore, development of automated scoring systems are desired. Its history is described in references ${ }^{1,2)}$. Presently, only a couple of groups are developing such systems for this purpose $\mathrm{e}^{3-5}$.

山本幹男：放射線医学総合研究所, 物理研究部, 千葉市稻毛区穴川4-9-1 宁263

TEL: $043-255-3139$

FAX: 043-251-9046

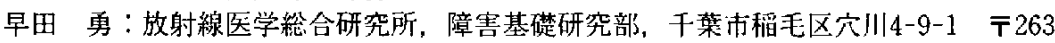

古田伸一：株式会社ニコン, 研究所, 品川区西大井1-6-3 テ 140 
Since 1989 we have promoted a project to develop an automated scoring system of radiation induced chromosome aberrations ${ }^{5-10}$ ). In this paper the project strategies are described. The sampling requirement for video (television) cameras, to achieve the best spatial resolution with an optical microscope ${ }^{11)}$, is considered ${ }^{6,7)}$. Choice of the high definition video camera is described. As a first step, a high resolution image processing system for study purposes, NIRS-1000: CHROMO STUDY, shown in Figs. 1 and 2, has been developed ${ }^{5-9)}$. It is tested that the system realizes close to the best spatial resolution utilizing a high definition video camera system. A preliminary algorithm, to classify chromosome aberrations, has been developed on the system using gray level information. Curved principal axis is taken with a new method. Small chromosomes are also treated by a new method. Preliminary test results for 10 excellent metaphases are shown.

\section{PROJECT STRATEGIES}

The final goal of our national project is the development of an automated scoring system of radiation induced chromosome aberrations. It is very important strategically to promote the project successfully, especially as in this case, over a short term and without enough grant and man power. Three of our strategies are as follows:

\section{Automation of specimen preparation}

In order to make automated image analysis easier, it is most important that the quality of specimens should be uniform and excellent for the automated analysis. Thus, it is the first strategy of our national project which includes two parts as follows:

The first part is the development of methods and the robot systems to prepare specimens with uniform and excellent quality for the automated image analysis. Preliminary work on the first part is described in other papers in this issue ${ }^{12,13)}$.

The second part is the development of methods and the image recognition system to score chromosome aberrations. The preliminary work on the second part is described in this paper.

It is assumed in this project that users use the combination of the specimen maker and the image analyzer. Therefore, we can avoid treating bad quality slides by the image analyzer.

\section{Two stage selections of metaphases}

It is shown in a paper ${ }^{14)}$ that the careful selection of analyzable metaphases has no effect on the scored ratio of the number of aberrations to the number of analyzed cells.

It is the second strategy that only selected excellent metaphases are treated by the image analyzer. The selection will be done in two stages.

In the first stage, metaphase finding and the selection of good metaphases will be done by NIRS-1000: METAPHASE RANKER as shown in Fig. 1 with a $\times 10$ objective lens.

In the second stage, the selection of excellent metaphases will be done by METAPHASE RANKER with a $\times 100$ objective lens.

A preliminary analysis of the second stage selection into "Excellnt" and "Others" has been 


\section{NIRS-1000: CHROMO STUDY}

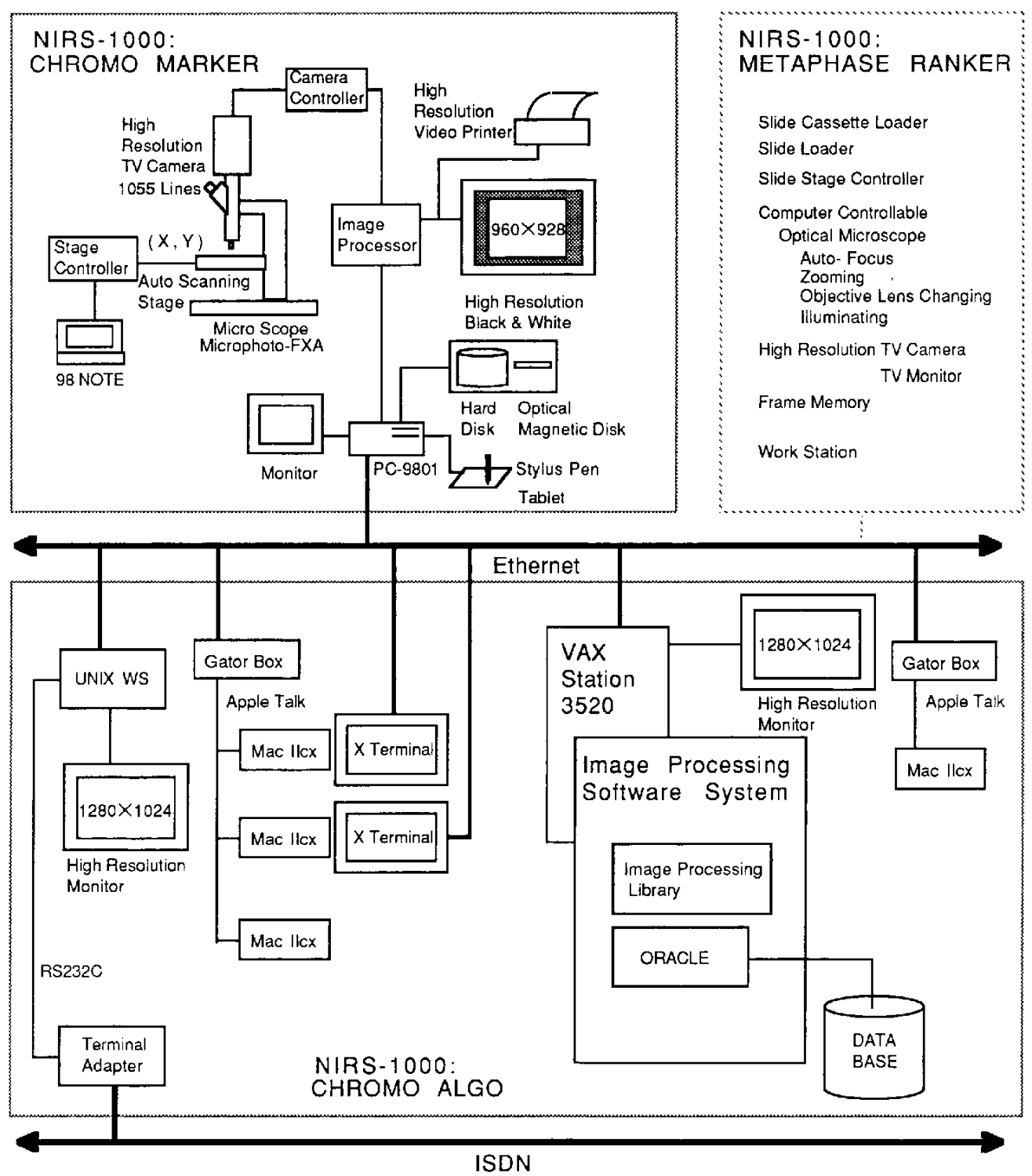

Fig. 1. NIRS-1000: CHROMO STUDY.

done with a simple algorithm for 50 metaphases. The test results show that the correctly classified ratio is $98 \%$, that the false positive is zero in this case, and that the false negative is $3 \%$. This algorithm and the test will be described elsewhere. 


\section{Best spatial resolution and maximum flexibility}

It is the third strategy that the project term should be divided into two stages: (1) a research and development study stage and (2) a development stage of practical systems.

In the first stage of the project, 1989-1994, a study purpose image processing system, NIRS-1000: CHROMO STUDY, will be developed. The design concept of the system is that the system should have the maximum flexibility and the best spatial resolution ${ }^{6,7)}$ rather than the processing speed.

In the second stage of the project, efforts will be made to speed up the system and to make the system economical. However, this stage has not yet been official.

\section{RESOLUTION LIMIT AND SAMPLING REQUIREMENT}

In this section, we consider the requirement of video cameras to realize enough vertical spatial sampling for achieving the best spatial resolution with a transparent optical microscope.

\section{Spatial resolution limit}

According to Rayleigh's Criterion ${ }^{11)}$, assuming that the optical system is ideal, the resolvable minimum distance, $d$, of two points is represented by equation (1).

$$
\mathrm{d}=0.61 \lambda / \mathrm{NA}
$$

Here, $\lambda$ is the wave length of incoherent light, and NA is the numerical aperture of an objective lens.

In our system described below, $\lambda=0.55 \mu \mathrm{m}$ and NA is 1.4 for the excellent $\times 100$ objective lens with oil immersion. Then $\mathrm{d}=0.24 \mu \mathrm{m}$. This is the theoretical limit of spatial resolution which is achievable in the system.

Even the power of eyepiece lenses and intermediate magnification lenses cannot help to achieve better resolution than this limit, because it is already defined by the objective lens.

\section{Sampling requirement}

According to the Sampling Theorem ${ }^{11)}$, the maximum allowable sampling interval, $s$, to achieve the spatial resolution, $d$, is about one half of the resolution. It is written as follow:

$$
\mathrm{s}=\mathrm{d} / 2 \text {. }
$$

Then the sampling interval should be less than $0.12 \mu \mathrm{m}$ to achieve the best resolution 0.24 $\mu \mathrm{m}$.

\section{CHOICE OF HIGH DEFINITION VIDEO CAMERAS}

The field of view of our microscope is $16 \mathrm{~mm}$ in diameter (Fig. 3). The effective size of imaging plates of $1^{\prime \prime}$ imaging tubes of our video cameras is about $10 \mathrm{~mm}$ in height and $13 \mathrm{~mm}$ in 


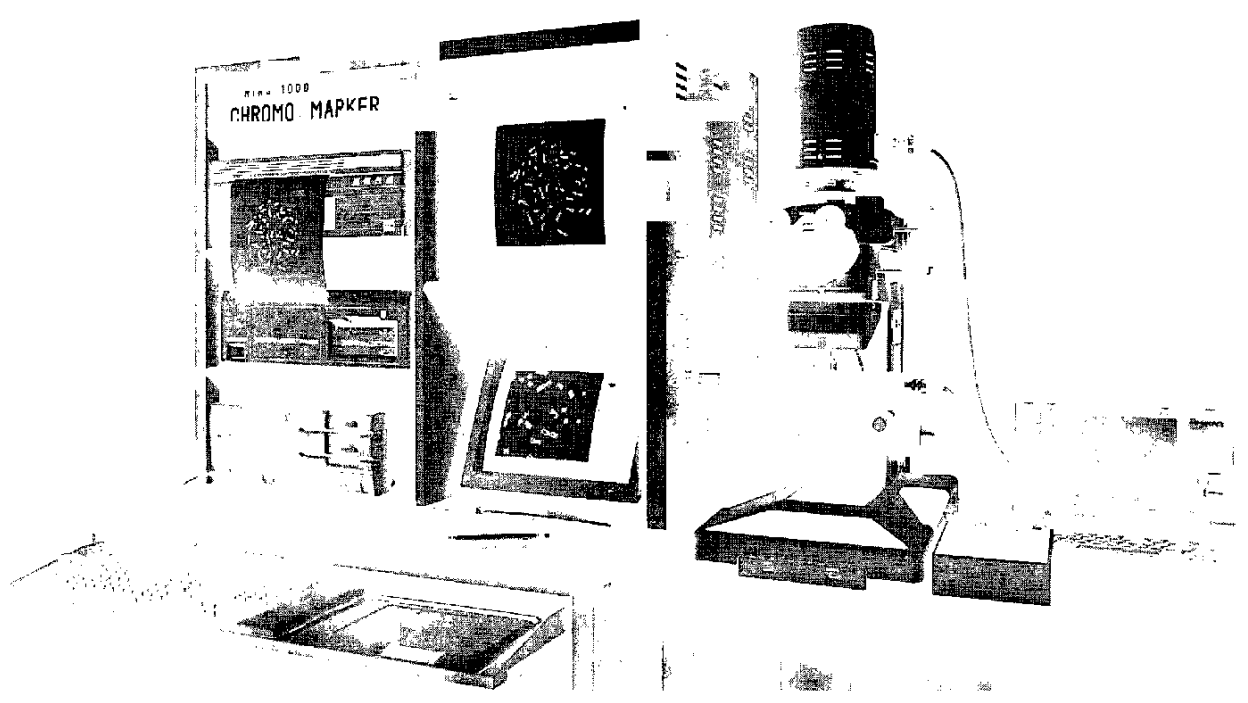

Fig. 2. NIRS-1000: CHROMO MARKER.

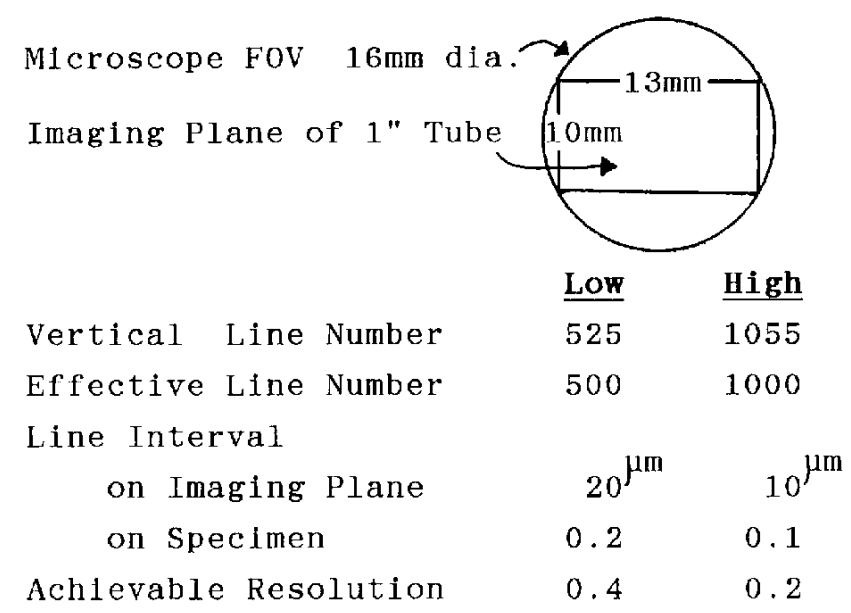

Fig. 3. Comparison of vertical sampling ability with conventional (Low) and high definition video cameras.

width. This means that the field of view on specimens has a height of $100 \mu \mathrm{m}$ when a $\times 100$ objective lens is used. This size is most adequate for our metaphase spread.

The NTSC-type conventional video camera has 525 scan lines and one type of high definition video camera has 1055 . We assume here that the effective lines are 500 for 
conventional cameras and $\mathbf{1 0 0 0}$ for high definition cameras for simplification of calculation.

This means that the sampling interval on imaging planes is $20 \mu \mathrm{m}$ for conventional cameras and $10 \mu \mathrm{m}$ for high definition cameras. Using the $\times 100$ objective lens, the realized sampling interval and the achievable best resolution on specimens are $0.2 \mu \mathrm{m}$ and $0.4 \mu \mathrm{m}$ respectively for conventional cameras, and $0.1 \mu \mathrm{m}$ and $0.2 \mu \mathrm{m}$ respectively for high definition cameras.

As a conclusion, it is impossible to achieve less than $0.4 \mu \mathrm{m}$ resolution with conventional cameras keeping the field of view on specimens as described above, while with enough sampling it is possible to achieve the best resolution, $0.24 \mu \mathrm{m}$, with the high definition cameras.

Therefore, we have chosen the high definition video cameras for this project. A test result of the achieved resolution on our system is shown in a later chapter.

\section{SYSTEM CONFIGURATION}

NIRS-1000: CHROMO STUDY is composed of three parts: (1) NIRS-1000: CHROMO MARKER $^{8,9)}$ (above the Ethernet in Fig. 1) whose main purpose is to mark on images to make image data base; (2) NIRS-1000: CHROMO ALGO (below) whose purpose is algorithm development; and (3) NIRS-1000: METAPHASE RANKER (above) whose purposes are metaphase finding and ranking with a low power objective lens, then ranking again with a high power objective lens. However METAPHASE RANKER is presently under development.

\section{NIRS-1000: CHROMO MARKER}

Main functions of CHROMO MARKER are stage control, high resolution image acquisition, putting marks easily on chromosome by an operator, scoring and making image data base. The TV system has 1055 scan lines to achieve $0.1 \mu \mathrm{m}$ sampling on specimens using an objective lens $(\times 100$, numerical aperture 1.4). The system resolution achieved on the hard copy is less than $0.3 \mu \mathrm{m}$ on specimens as shown in the next chapter.

\section{System configuration}

System configuration of NIRS-1000: CHROMO MARKER is shown in Fig. 1. The system can be divided into five parts described as follows: a) an optical microscope, b) a stage control, c) high resolution imaging, d) processing, and e) a data base. The system shape except the image processor is shown in a photograph (Fig. 2).

a) The optical microscope is a conventional model with the highest functions, MicrophotoFXA made by Nikon. The functions are as follows: (1) Four ports for photographic and video cameras. These are convenient for research purpose. (2) An electrically driven revolver for changing objective lens. (3) A conventional autofocus device can also be built in. However the autofocus is out of the specification and does not work well for chromosome studies with high power $(\times 100)$ objective lenses. Thus a new method is under development for NIRS-1000: METAPHASE RANKER. 
b) The stage control part is composed of a two dimensional microscope stage driven with a pulse motor $(025 \mu \mathrm{m} / \mathrm{step})$, a stage control electronic unit, and a personal computer (NECPC98note)

c) The high resolution imaging part is composed of a high definition video camera, frame memories which are in an image processor, a black and white video monitor and a video hard copy printer The video camera is made by Hamamatsu Photonics, viz High Reso Camera C2397, with a $1^{\prime \prime}$ special Vidicon type tube having 1055 scan lines The effective imaging plane size is approximately $13 \mathrm{~mm}$ horizontally and $10 \mathrm{~mm}$ vertically The image processor is a PIAS-made LA-1000 It consists of a 8 bit analog-to-digital converter for image intensity, a XY position digitizer for video position signal, four $1024 \times 1024 \times 8$ bit frame memories, simple hard processors and a CPU The black and white high definition video monitor is DMA-153 made

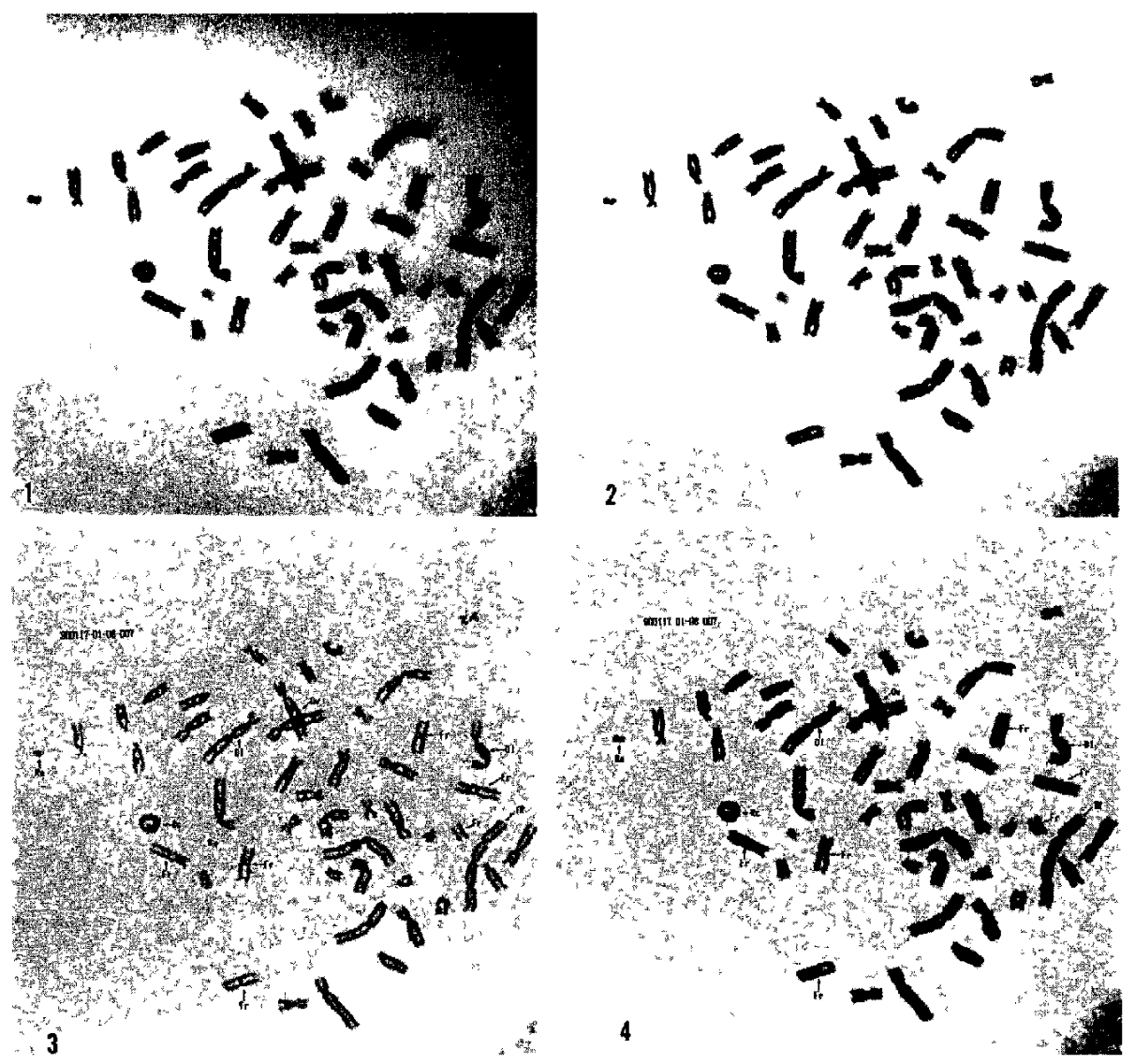

Fig. 4. Hard copy images output from NIRS-1000 CHROMO MARKER

(1) A metaphase chromosome image

(2) After back ground subtraction

(3) Marked umage similar to Fig 5

(4) Marked image without chromosome mark or centromere mark 
by Ikegami. The effective scan line is 1024 , and the maximum number of horizontal display pixels is 1024. The video hard copy printer is Seiko-made VP-3500. It is a thermal printer, and the specifications are $1024 \times 1024$ pixels, 64 gray levels, and 300 dots per inch.

d) The processing part is composed of a NEC-made personal computer PC-9801, a stylus pen

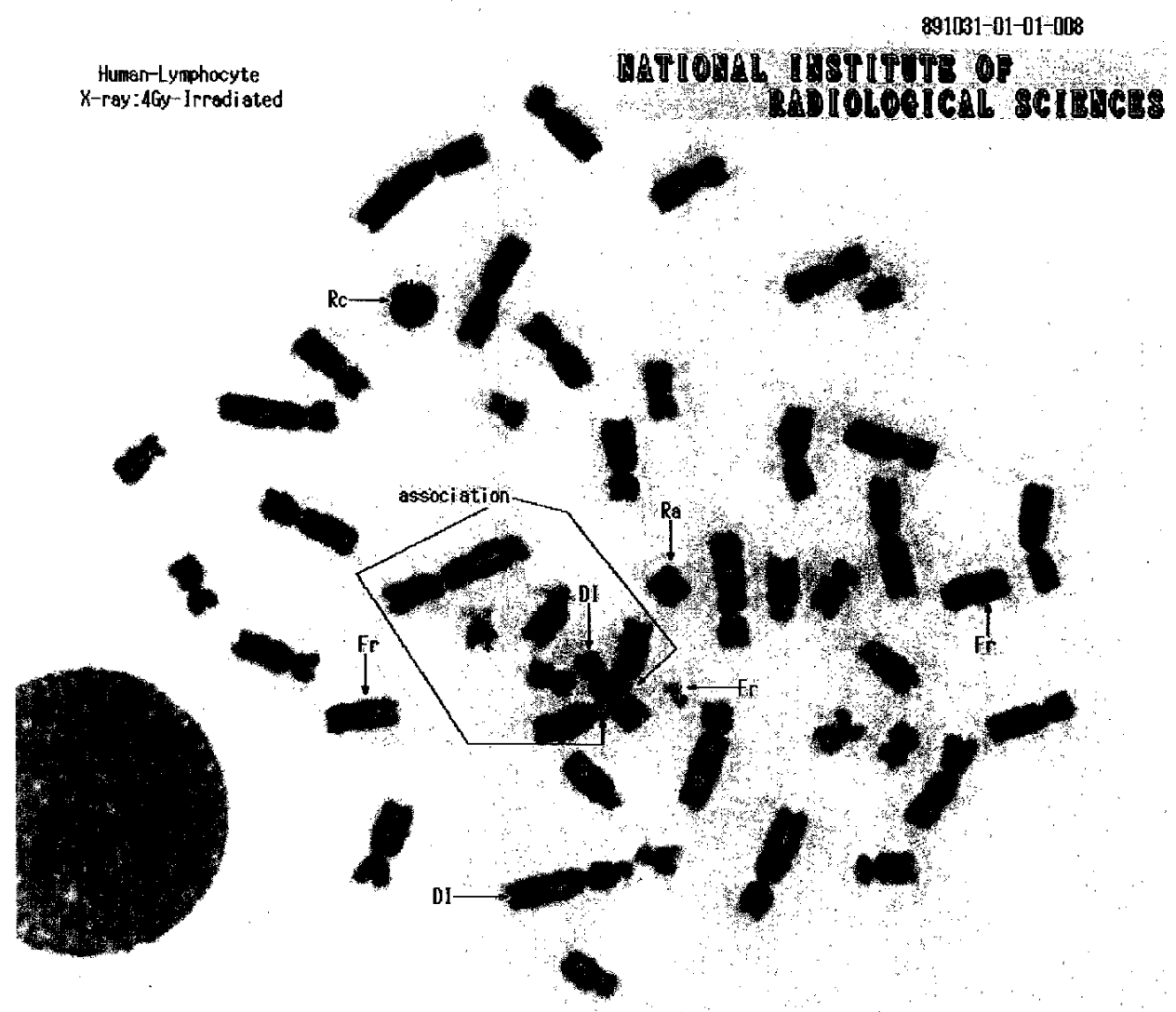

Fig. 5. A hard copy of the marked image with NIRS-1000: CHROMO MARKER.

A metaphase chromosome image of the human peripheral lymphocyte irradiated by $\mathrm{x}$-ray of $4 \mathrm{~Gy}$.

*: Chromosome of fragment.

+ : Centromere position.

DI: Dicentric.

Ra: Acentric ring.

Rc: Centric ring.

Fr: Fragment.

Ov: Overlapped. 
and a tablet.

c) The data base part is composed of a hard disk and a cassette type optical magnetic disk.

\section{Main functions}

a) The stage of the microscope is steered electronically in two dimensions by steps of $0.25 \mu \mathrm{m}$ step with a joy-stick. Stored locations are automatically relocated by a personal computer.

b) Microscopic images are taken by a high definition video camera with 1055 scan lines. The effective image size is $960(\mathrm{H}) \times 928(\mathrm{~V})$ pixels, and the gray scale is 256 levels.

c) The images are processed by an image processor and a personal computer, and these are displayed on a high resolution black and white monitor.

d) An operator can easily mark the positions of chromosomes, using abbreviated letters of choice for all kinds of chromosome aberrations, centromere positions, etc. with a stylus pen on a tablet; comments can be entered with a key board (Figs. 4 and 5).

e) Simultaneously with the marking, scores corresponding to each kind of aberration, number of centromeres, number of chromosomes, etc. are automatically increased.

f) Semi-automated chromosome karyotyping is available ${ }^{15)}$.

g) Both the image data and the marking data are stored in cartridge type optical magnetic disks
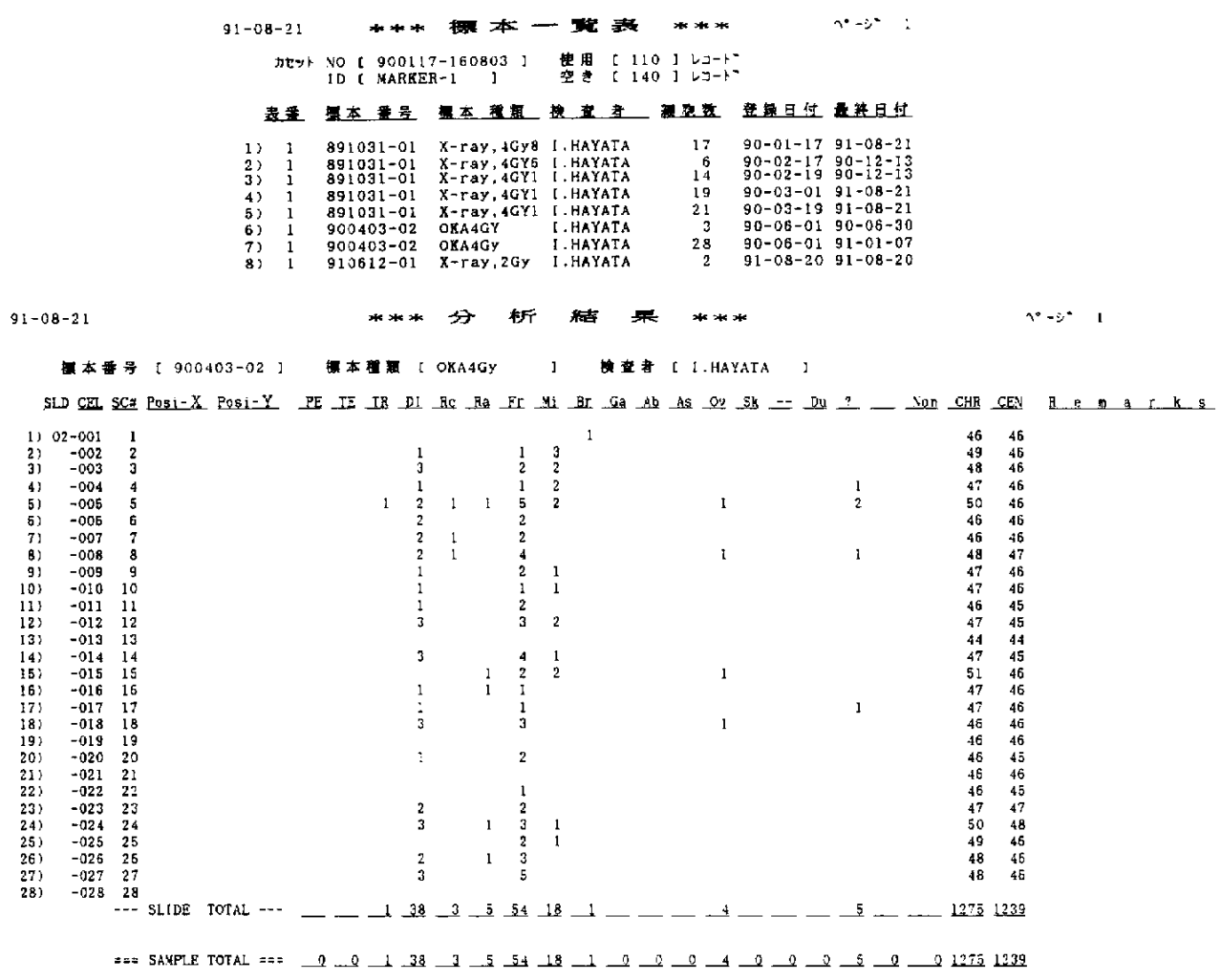

Fig. 6. Example of analyzed summary of chromosome aberrations induced by radiations. 
(each has 650 Mbytes).

h) High quality image hard copies (64 gray levels) are easily obtained in a few seconds (Fig. 5). The related reports and the score tables are printed out (Fig. 6).

i) The data are transferred to a VAX station computer system through Ethernet for data base.

\section{SPATIAL RESOLUTION TEST}

The system spatial resolution of NIRS-1000: CHROMO MARKER was tested. A tiny Fresnel zone plate on a specimen slide was used as a test pattern. The outermost ring width is

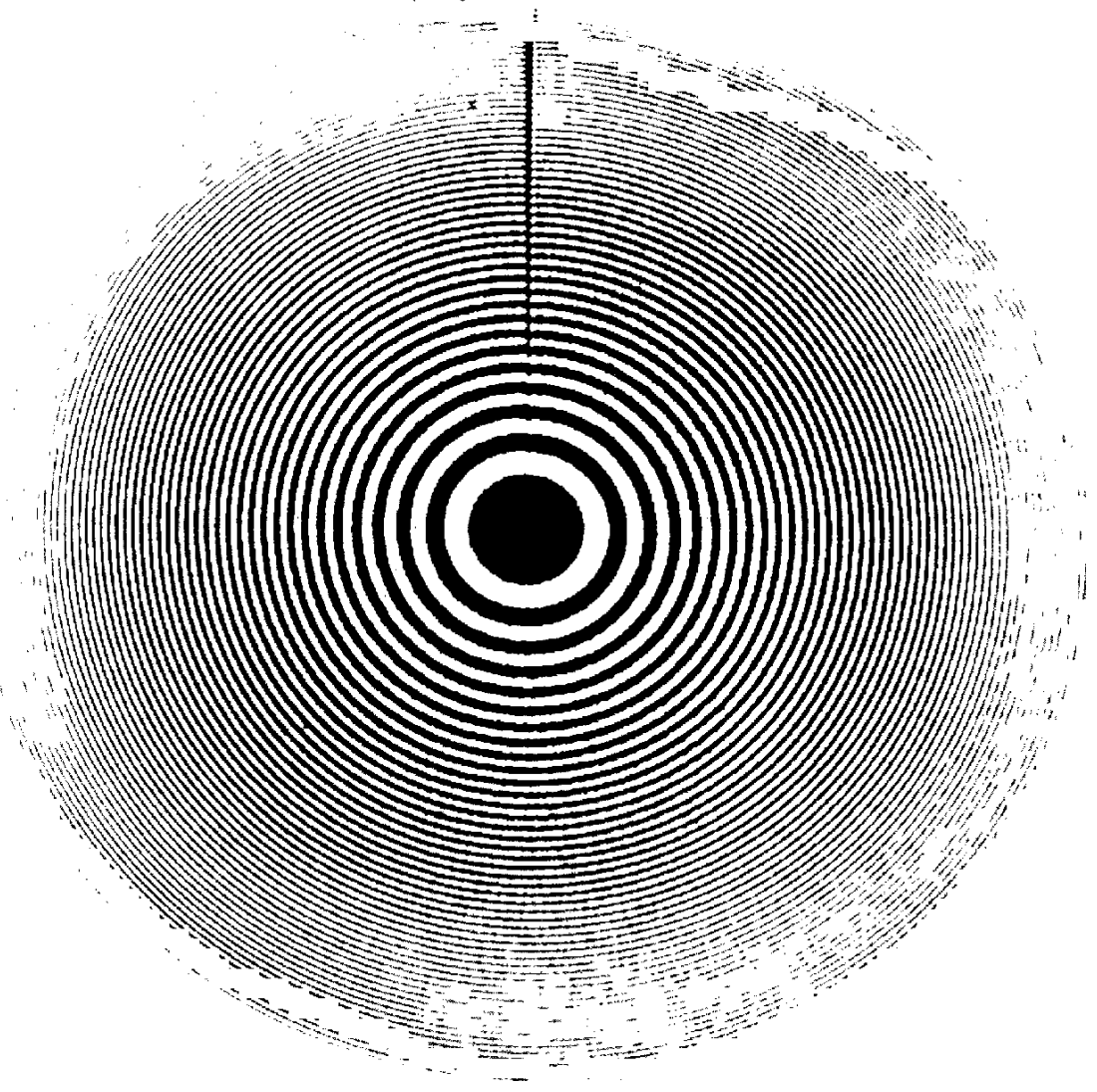

Fig. 7. System spatial resolution test of NIRS-1000: CHROMO MARKER. A hard copy image of a tiny Fresnel zone plate on a specimen slide. The outermost ring width is $0.25 \mu \mathrm{m}$ which is very close to the theoretical resolution limit of the optical microscope, $0.24 \mu \mathrm{m}$. This experiment shows that at least $0.3, \iota \mathrm{m}$ is resolved even on the hard copy of this system. 
$0.25 \mu \mathrm{m}$ which is very close to the theoretical resolution limit of the optical microscope, $0.24 \mu \mathrm{m}$. The high power objective lens ( $\times 100$, Nikon NCF Plan Apo) and an excellent condenser lens (Achromatic Aplanat), both with numerical aperture of 1.4 , were used with oil immersion without additional intermediate magnification.

Fig. 7 shows that at least $0.3 \mu \mathrm{m}$ is resolved even though on the hard copy output of the system no image processing was applied. In the last chapter the existence of a small difference between the theoretical limit and the value achieved has been discussed.

\section{NIRS-1000: CHROMO ALGO}

CHROMO ALGO is composed of a computer system shown in Fig. 1 and an original software system for image processing, on which automated recognition algorithms are studied. CHROMO ALGO has three functions which are respectively, organizing image analysis, data base and data transport.

\section{Hardware}

CHROMO ALGO consists of a VAX Station 3520 and an UNIX work station. Each of these is connected by Ethernet and is able to transport data using TCP/IP protocol. Macintosh and $X$-terminals are used as terminals for these computers via ethernet. The work stations have $1280 \times 1024$ color monitors and X-terminals have $1024 \times 768$ color monitors which are able to display the chromosome images with 256 gray levels using $X$-Window System.

\section{Software}

We have developed a program system called Platform which is able to organize image analysis and construct data base on VAX Station. This program is written in C language and uses a X-Window System.

Platform receives the image data and some additional information from CHROMO MARKER via ethernet and constructs data base using ORACLE, a relational data base software. We can use this data base through Platform easily.

To analyze the image, considerable amount of work is required. These are composed of two parts; one is creating small subroutines which treat the image, and the other is combining these subroutines. The latter work is more complicated. Platform offers the environment within which we can combine these subroutines freely.

\section{ALGORITHM TO CLASSIFY ABERRATIONS}

\section{Purpose of algorithm}

A preliminary algorithm developed on NIRS-1000: CHROMO STUDY is described in this section. The purpose of the algorithm is automated classification of chromosomes into aberration classes: tricentric, dicentric, ring, fragment, and normal. This is the preliminary study of 
the final goal to score radiation induced chromosome aberrations.

\section{Objective metaphase images}

It is assumed that only selected excellent metaphases for automatic analysis are treated with the algorithm below, such as described in Project Strategies section.

Therefore, chromosomes in the object metaphases are in the phase when the chromatides are clearly separated, but are connected at the centromere. An example of object metaphase is shown in Fig. 8. The metaphases are stained with conventional Giemsa. The images were taken with CHROMO MARKER and were stored as the image data base. The images have 256 gray levels, and the size is $960 \times 928$ pixels.

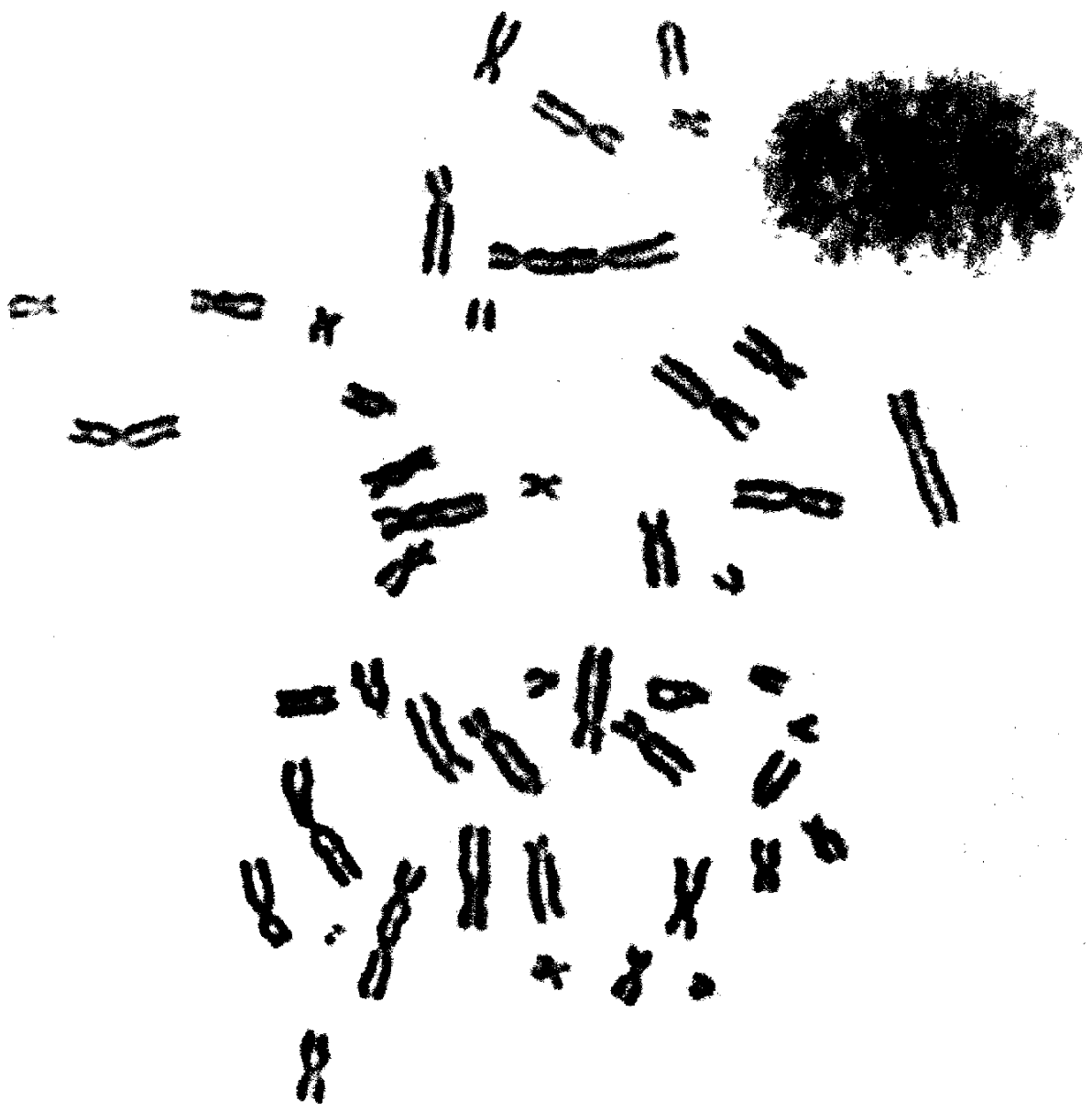

Fig. 8. An example of analyzed metaphase spread of human peripheral lymphocyte irradiated by $\mathrm{x}$-ray of $4 \mathrm{~Gy}$. 


\section{Classification Flow of Aberrations}

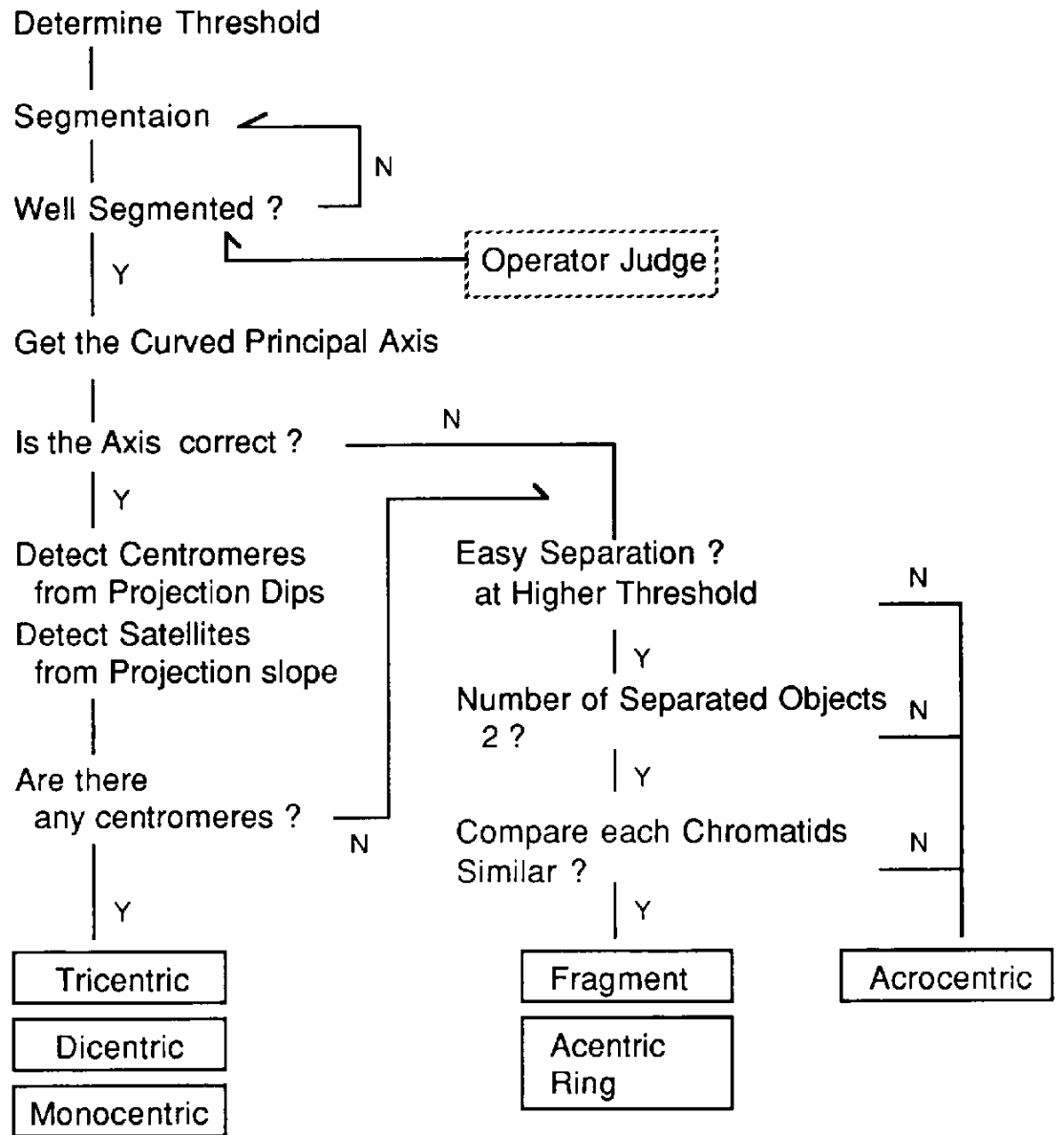

Fig. 9. Flow diagram to classify the chromosomes.

\section{Flow diagram}

The flow diagram of the algorithm, to classify the chromosomes shown in Fig. 9, is as follows:

1) Temporal automatic segmentation

This determines the threshold gray level iteratively to binarize the global image for the temporal segmentation so that the number of segmented objects is 46 . Although this method gives a good threshold level it takes a lot of time. Fig. 10 shows an example of temporally automatic segmented images of the metaphase spread obtained from Fig. 8. It includes one touched chromosome and one nucleus.

2) Semi-automated segmentation

It displays the result of temporal segmentation (Fig. 10). An operator assigns improperly 


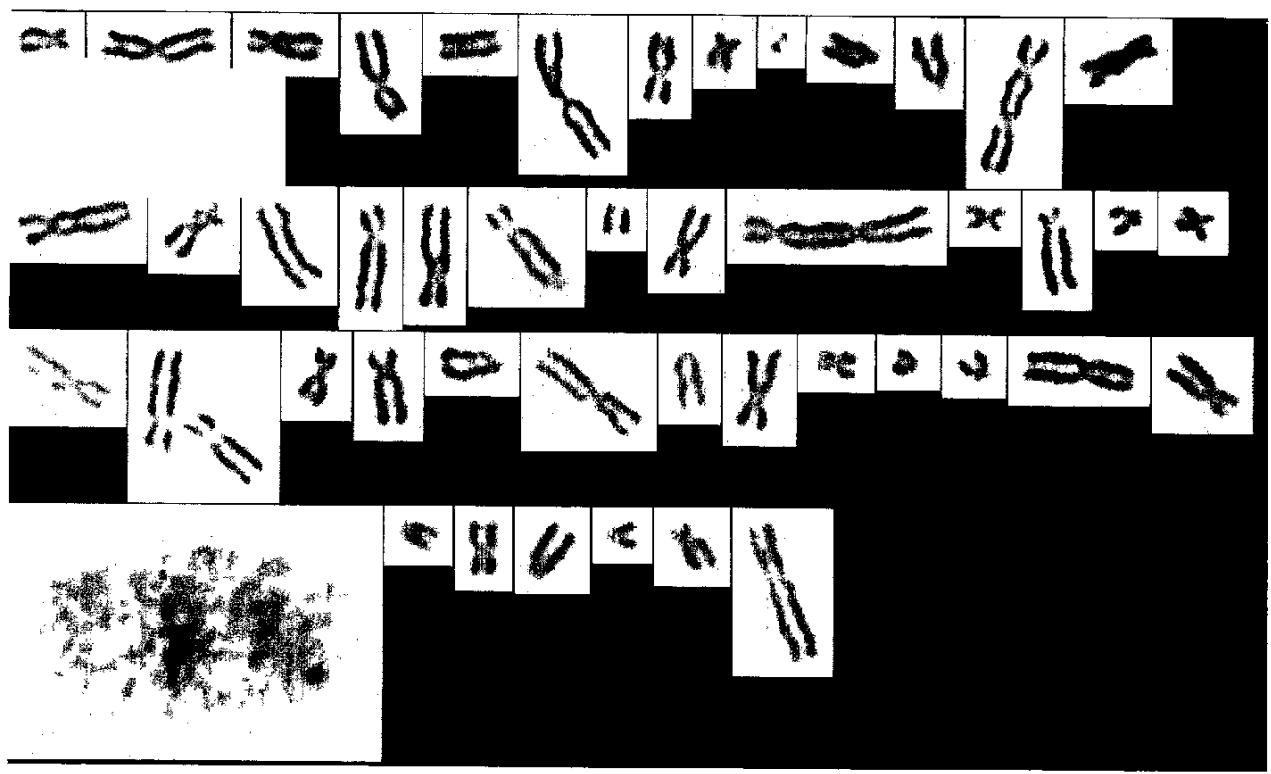

Fig. 10. Result of temporal automatic segmentation of the metaphase shown in Fig. 8 . It includes one touched chromosome and one nucleus. The touched one will be separated automatically into two objects after assignment by an operator for a touched one.

segmented objects as a nucleus, or a touched chromosome and others. Once a touched chromosome is assigned, it is automatically separated into two parts by trying higher threshold levels only for the touched chromosome part.

We intend to make an entire segmentation process fully automatic in the near future.

3) Principal axis and projection

A principal axis is obtained for a segmented chromosome. Then one gets the projection across the principal axis, if this axis is correct.

The projection here means a plot of the integrated pixel gray values perpendicular to the principal axis. It shows well the locations of the centromeres as dips; an example is shown in Fig. 11(f) ${ }^{16.17)}$

There are several methods to obtain the principal axis ${ }^{17}$. The simplest one is the straight line given by a calculation of the moment of each pixel gray value in the chromosome. This line corresponds well to the principal axis when the chromosome is straight. On the contrary, when the chromosome is bent, it is insufficient that this line is regarded as the correct principal axis.

As shown in Fig. 11 the chromosome (c) is bent dicentric; on graph (d) there is one obvious dip; the chromosome (e) is the straightened image of (c) by the curved principal axis; and on graph (f) there are two obvious dips, each dip indicating the centromere well.

If we want to determine the centromere location from the projection, it is necessary to get the correct principal axis which is curved along the chromosome for bent chromosomes. The method will be explained in a section below.

We may judge whether the principal axis is correct or not by obtaining a projection whose 


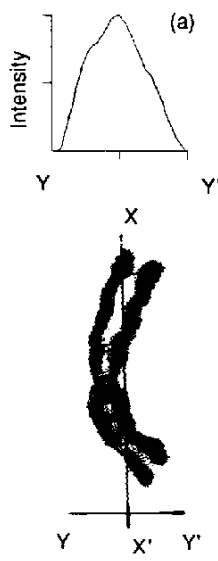

(c)

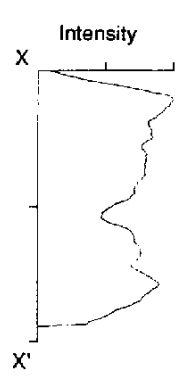

(d)
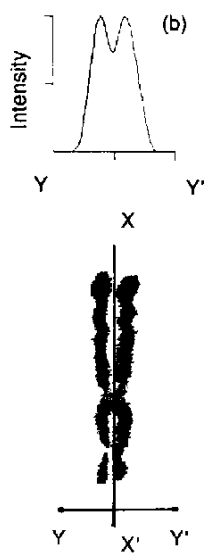

(e)

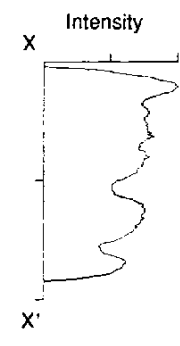

(f)

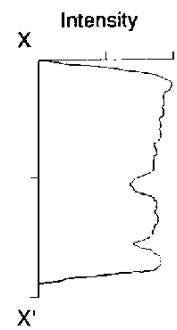

(g)

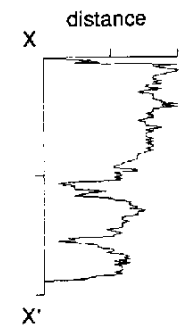

(h)

Fig. 11. Comparison of a linear principal axis and a curved principal axis.

(a) (b) Projection parallel to the principal axis.

(c) Bent chromosome which has two centromeres.

(d) (f) Projection across the principal axis.

(e) Straightened image of (c) by the curved principal axis.

(g) Pixel values on the chromatid ridges.

(h) Distance between two chromatid ridges.

integration is done parallel to the principal axis. If it is correct, the projection has two peaks, i.e. bimodal, as shown in Fig. 11(b). If it is incorrect, the projection has only one peak as shown in Fig. 11(a).

4) Detect centromeres

The centromeres are detected from the dips of the projection (Fig. 11(f)) whose value is below the threshold value of intensity (Fig. 12(a), (b), (c)). The centromeres, which are located at the end of the chromosomes (Fig. 12(d)), are detected from the shape of the slope of each end of the projection.

5) Classification

The chromosomes utilization of the number of centromeres is classified. If the chromosome has no centromeres, or if the principal axis is incorrect, then, go to 'For a small chromosome' (Fig. 12(e)).

6) For a small chromosome

Generally, for small chromosomes, it is difficult to get the principal axis. Thus, we have developed a method to classify the small chromosomes without getting the principal axis.

The threshold gray level is increased to binarize the small chromosome. Then the chromosome separates into a few objects which might be chromatids as shown in Fig. 13 . When the chromosome is separated, we can classify this chromosome by the number of separated objects, by the ease of separation and by comparing the chromatids. In the case of a fragment (Fig. 13(c)), it is separated into two objects easily, and the separated objects are similar to each other. 

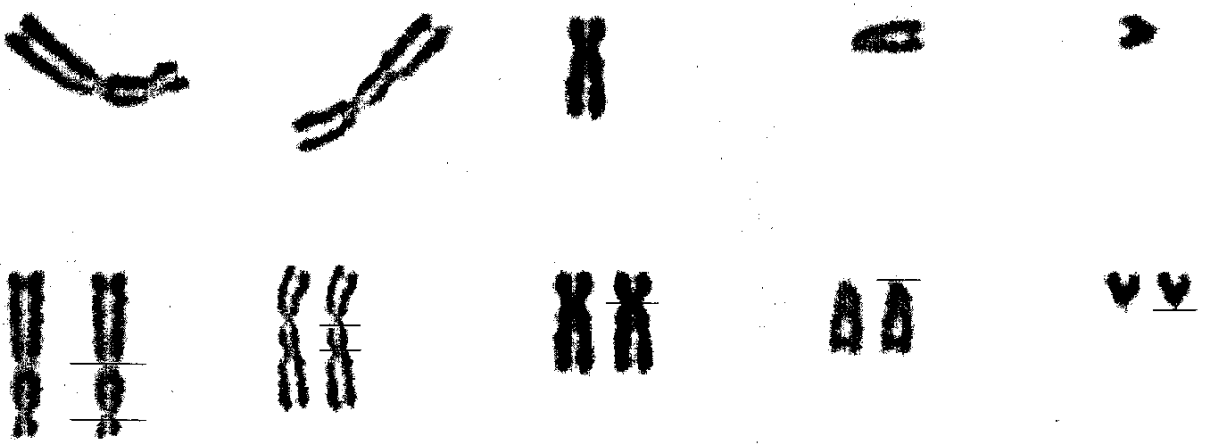

(a)

(b)

(c)

(d)

(e)

Fig. 12. Some successful samples of detected centromere locations (shown by horizontal line in the chromosome image). Upper ones are original images, lower ones are straightened, and rotated images.

(a) (b) Dicentric.

(c) Monocentric.

(d) (e) Acrocentric.

(a)
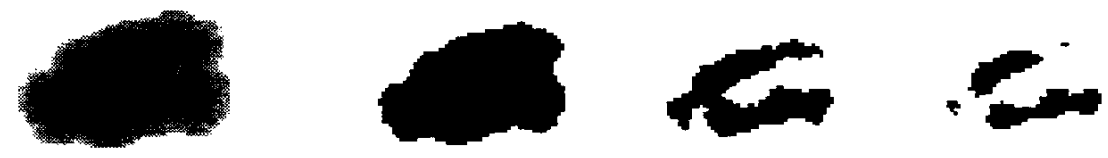

(b)
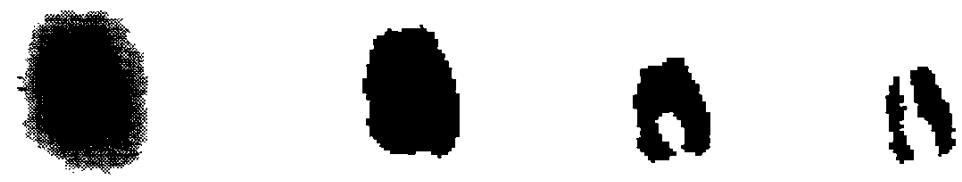

(c)
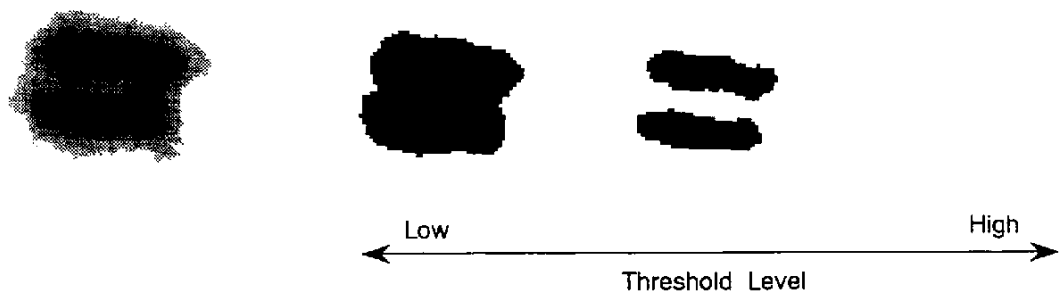

Fig. 13. Chromosomes with changing threshold levels. Left column shows gray level images. The others are binary images. Right column is higher level.
(a) Acrocentric (D group).
(b) Acrocentric (Y).
(c) Fragment. 
In Fig. 12, there are some successful examples of detecting the locations of the centromeres shown with the horizontal lines in the image.

\section{Curved principal axis}

A new method to get a curved principal axis is explained in this section.

The linear principal axis $X-X^{\prime}$ is obtained by the moment method (Fig. 14(a)). Then the profile (Fig. 14(b)) across the principal axis $\mathrm{Y}^{-Y^{\prime}}$ is determined. If the profile has two peaks (Fig, 14(b)), the middle point between these is plotted and shifted along the principal axis (Fig. 14(c)). Finally these points are connected by lines, and the lines are extrapolated at the each end (Fig. 14(d)).

\section{Principal Axis}

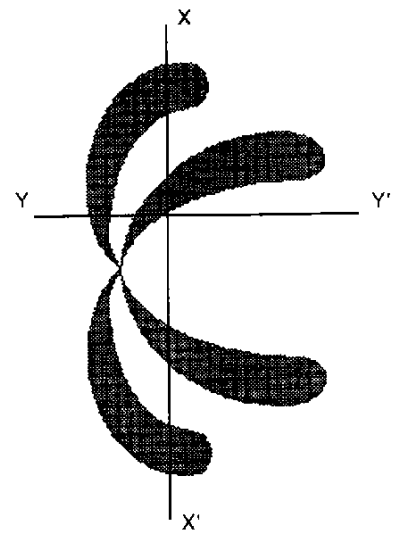

(a)

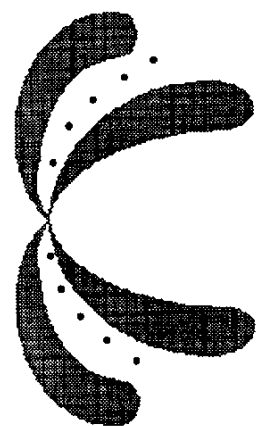

(c)

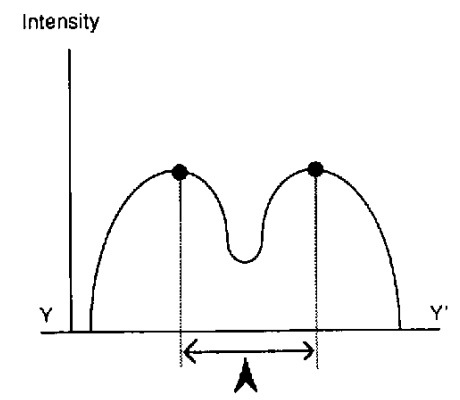

(b)

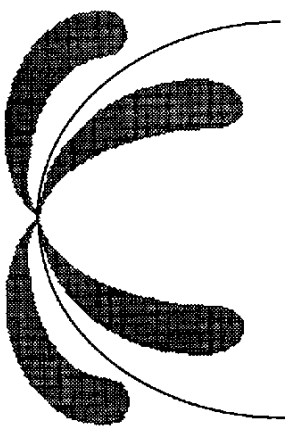

(d)

Fig. 14. Procedure to get the curved principal axis.

(a) Obtain the linear principal axis X-X' by the moment method.

(b) Density profile across the principal axis $Y-Y$ '. Fixed circles show two maxima, upper arrow shows the middle point between the two maxima.

(c) Plot the middle points between these two peaks.

(d) Connect these points by some lines, and extrapolate at each end. 
An example of this method to a dicentric chromosome is shown in Fig. 15. The original image is shown in (a). The linear principal axis is shown in (b). The linear pricipal axis is rotated so as to be vertical as shown in (c). This image is inferior to the original one because of lack of interpolation. The middle points between two chromatids are plotted as shown in (d). These points are connected by lines and extrapolated at each end as shown in (e). The image is straightened by the curved principal axis as shown in (f).

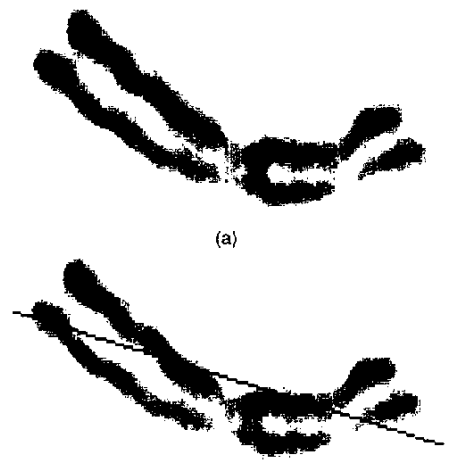

(b)

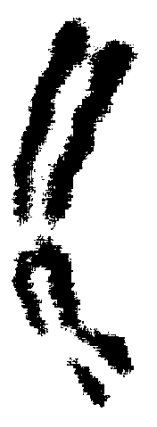

(c)

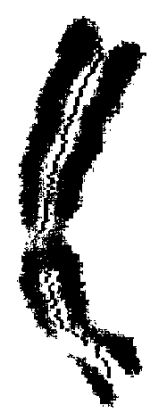

(d)

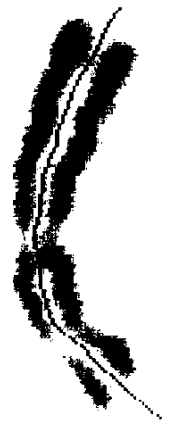

(e)

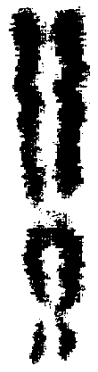

(1)

Fig. 15. An example for applying this method to a chromosome.

(a) Original image.

(b) Get the principal axis by the moment.

(c) Rotate as the principal axis is vertical.

(d) Plot the middle points.

(e) Connect the points and extrapolate at each end.

(f) Straightened with this curved principal axis.

\section{TEST RESULTS OF ALGORITHM}

Test results of this program, applied to the selected excellent metaphases irradiated by $4 \mathrm{~Gy}$ $\mathrm{X}$-rays, are summarized as the confusion matrices in Table 1 to 7 . The program has been trained with 5 metaphases (Table 1 and 2). Then, it was applied to 5 additional new metaphases (Table 3 and 4). The total of these 10 metaphases are shown in Table 5,6 and 7.

Table 1, 3 and 5 show chromosomes classified into four classes: tricentric, dicentric, fragment and monocentric. Rings are not included in the 10 metaphases which were tested. Table 2, 4 and 6 summarize Table 1, 3 and 5 respectively by grouping of the three classes, tricentric, dicentric and fragment, as aberrations. Table 7 shows chromosomes calssified into three groups, non-acrocentric, acrocentric and fragment, according to the location of centromeres for the ten metaphases. 
Table 1. Chromosomes classified into four classes on five metaphases trained

\begin{tabular}{rlccccr}
\hline \multirow{2}{*}{ Computer } & \multicolumn{5}{c}{ Human } \\
\cline { 5 - 7 } & Class & Tricentric & Dicentric & Fragment & Monocentric & Total (\%) \\
\hline \multirow{3}{*}{ Tricentric } & 1 & 1 & & 3 & $5(20.0)$ \\
& Dicentric & & 11 & & 4 & $15(73.3)$ \\
& Fragment & & & 15 & 6 & $21(71.4)$ \\
& Monocentric & & 1 & 2 & 188 & $191(98.4)$ \\
\hline & Total (\%) & $1(100)$ & $13(84.6)$ & $17(88.2)$ & $201(93.5)$ & $232(92.7)$ \\
\hline
\end{tabular}

Table 2. Chromosomes classified into two classes on five metaphases tested

\begin{tabular}{ccccccc}
\hline & \multicolumn{5}{c}{ Human } \\
\hline \multirow{3}{*}{ Computer } & Aberrations & 28 & TP $(90.3 \%)$ & 13 & FP $(6.5 \%)$ & $41(68.3 \%)$ \\
\cline { 2 - 7 } & Monocentric & 3 & FN $(9.7 \%)$ & 188 & TN $(93.5 \%)$ & $191(98.4 \%)$ \\
\hline & Total & 31 & $(90.3 \%)$ & 201 & $(93.5 \%)$ & $232(93.1 \%)$ \\
\hline
\end{tabular}

Aberrations includes Tricentric, Dicentric and Fragment in Table 1.

TP: true positive, FP: false positive,

$\mathrm{FN}$ : false negative, $\mathrm{TN}$ : true negative.

Table 3. Chromosomes classified into four classes on five metaphases applied

\begin{tabular}{|c|c|c|c|c|c|c|}
\hline \multicolumn{7}{|c|}{ Human } \\
\hline & Class & Tricentric & Dicentric & Fragment & Monocentric & Total $(\%)$ \\
\hline \multirow{5}{*}{ Computer } & Tricentric & & & & 1 & $1(0.0)$ \\
\hline & Dicentric & & 7 & & 7 & $14(50.0)$ \\
\hline & Fragment & & & 10 & 4 & $14(71.4)$ \\
\hline & Monocentric & & 3 & 3 & 197 & $203(97.0)$ \\
\hline & Total (\%) & & $10(70.0)$ & $13(76.9)$ & $209(94.3)$ & $232(92.2)$ \\
\hline
\end{tabular}

Table 4. Chromosomes classified into two classes on five metaphases applied

\begin{tabular}{ccccccc}
\hline & \multicolumn{5}{c}{ Human } \\
\hline \multirow{3}{*}{ Computer } & Class & \multicolumn{2}{c}{ Aberrations } & Monocentric & Total \\
\cline { 2 - 7 } & Aberrations & 17 & TP $(73.9 \%)$ & 12 & FP $(5.7 \%)$ & $29(58.6 \%)$ \\
\hline & Monocentric & 6 & FN $(26.1 \%)$ & 197 & TN $(94.3 \%)$ & $203(97.0 \%)$ \\
\hline
\end{tabular}

Aberrations includes Tricentric, Dicentric and Fragment in Table 3. 
Table 5. Chromosomes classified into four classes on ten metaphases

\begin{tabular}{clccccr}
\hline \multirow{2}{*}{ Class } & Tricentric & Dicentric & Fragment & Monocentric & Total (\%) \\
\hline \multirow{3}{*}{ Computer } & Tricentric & 1 & 1 & & 4 & $6(16.7)$ \\
& Dicentric & & 18 & & 11 & $29(62.1)$ \\
& Fragment & & & 25 & 10 & $35(71.4)$ \\
& Monocentric & & 4 & 5 & 385 & $394(97.7)$ \\
\hline & Total $(\%)$ & $1(100)$ & $23(78.3)$ & $30(83.3)$ & $410(93.9)$ & $464(92.5)$ \\
\hline
\end{tabular}

Table 6. Chromosomes classified into two classes on ten metaphases

\begin{tabular}{ccccccc}
\hline & \multicolumn{5}{c}{ Human } & \\
\hline \multirow{3}{*}{ Computer } & Class & \multicolumn{2}{c}{ Aberrations } & \multicolumn{2}{c}{ Monocentric } & \multicolumn{1}{c}{ Total } \\
\cline { 2 - 7 } & Aberrations & 45 & $\mathrm{TP}(83.3 \%)$ & 25 & $\mathrm{FP}(6.1 \%)$ & $70(64.3 \%)$ \\
\cline { 2 - 8 } & Monocentric & 9 & $\mathrm{FN}(16.7 \%)$ & 385 & $\mathrm{TN}(93.9 \%)$ & $394(97.7 \%)$ \\
\hline & Total & 54 & $(83.3 \%)$ & 410 & $(93.9 \%)$ & $464(92.7 \%)$ \\
\hline
\end{tabular}

Aberrations includes Tricentric, Dicentric and Fragment in Table 5.

Table 7. Chromosomes classified by the location of centromeres on ten metaphases

\begin{tabular}{|c|c|c|c|c|c|}
\hline \multicolumn{6}{|c|}{ Human } \\
\hline & group & $\begin{array}{c}\text { Non-Acrocentric } \\
\text { A, B, C, E, F group }\end{array}$ & $\begin{array}{c}\text { Acrocentric } \\
\mathrm{D}, \mathrm{G} \text { group and } \mathrm{Y}\end{array}$ & Fragment & Total $(\%)$ \\
\hline \multirow{4}{*}{ Computer } & $\begin{array}{l}\text { Non-Acrocentric } \\
A, B, C, E, F \text { group }\end{array}$ & 305 & 4 & & 309 (98.7) \\
\hline & $\begin{array}{l}\text { Acrocentric } \\
D, G \text { group and } Y\end{array}$ & 35 & 101 & 5 & $141(71.6)$ \\
\hline & Fragment & 3 & 7 & 25 & $35(71.4)$ \\
\hline & Total $(\%)$ & $343(88.9)$ & $112(90.2)$ & $30(83.3)$ & $485(88.9)$ \\
\hline
\end{tabular}

\section{DISCUSSIONS AND CONCLUSIONS}

The final goal of our national project is the development of an automated scoring system for radiation induced chromosome aberrations.

The project strategies are as follows. (1) Development of an automation for specimen preparation as well as for image recognition. (2) Two stage selection of metaphases which will follow. The second stage selection will be done with a $\times 100$ objective lens to select only excellent metaphases for an image analyzer. Items (1) and (2) will make the image recognition 
easier. (3) To start from a system with the best possible spatial resolution and maximum flexibility.

Theoretical spatial resolution limit of optical microscope with an objective lens of 1.4 numerical aperture is $0.24 \mu \mathrm{m}$. The sampling requirement to achieve this limit is a maximum of $0.12 \mu \mathrm{m}$ interval. Vertical sampling with 500 scan lines is $0.2 \mu \mathrm{m}$ and with 1000 lines is $0.1 \mu \mathrm{m}$. Therefore, we chose a high definition video camera for our system described below.

NIRS-1000: CHROMO STUDY has been developed as a high resolution image processing system for study purposes. It consists of CHROMO MARKER, CHROMO ALGO and CHROMO RANKER.

CHROMO MARKER gives us high resolution microscopic digital images and their hard copies. It has been tested that, even on the hard copies, $0.3 \mu \mathrm{m}$ on specimens is resolved when a high power objective lens $(\times 100$, numerical aperture 1.4$)$ is used with oil immersion and without any additional intermediate magnification and without any image processing.

There is a small difference between the theoretical limit and the achieved resolution. This is reasonable, because of the beam spot size for the video camera reading, aberrations of optical lenses, insufficient line number of the hard copy device and improper interpolation processing.

We can easily put a mark of each chromosome aberration, centromere position, etc. on the image. The results are automatically scored and printed out.

It is proven by experience of a year and a half that CHROMO MARKER is useful for making image data base, routine analysis, and for educational purposes.

CHROMO ALGO is a computer system with original software system on which algorithms for automatic chromosome recognition have been studied and developed comfortably.

CHROMO RANKER is a combination of mechanical, optical and computer system on which metaphase finding with a low power objective lens, and metaphase ranking with a high power $(\times 100)$ objective lens will follow. The hardware has been developed and the software is being developed now. Details of the system will be reported elsewhere.

A preliminary algorithm has been developed on NIRS-1000: CHROMO STUDY. The purpose is automated classification of chromosomes in selected excellent metaphases into classes of aberration, tricentric, dicentric, ring, fragment, and normal. This is the first stage of study of the final goal to score radiation induced chromosome aberrations. The algorithm uses projection information of gray level and easiness of separation information for small chromosomes.

The preliminary test results on selected excellent 10 metaphases show that the correct classification ratio for four classes is $92.5 \%$ (Table 5) and the ratio for two classes is $92.7 \%$. The detection rate of the aberrations is $83.3 \%$ and the false positive rate is $6.1 \%$ (Table 6). The correct classification ratio for three classes, non-acrocentric, acrocentric, and fragment, is $88.9 \%$ (Table 7). Detection ratios of the three classes are $88.9 \%, 90.2 \%$, and $83.3 \%$, respectively.

It is our intention to improve the algorithm to introduce more information such as the profiles along chromatid's ridge (Fig. $1 \mathrm{l}(\mathrm{g})$ ) and the distance between the chromatid's ridge (Fig. 11(h)), to make fully automatic segmentation, and to test the algorithm on many more metaphases in the near future. 


\section{ACKNOWLEDGEMENTS}

This work has been promoted as a project of Assessment and Reduction of Radiation Risks, which belongs to Cross-Over Research on Underlying Technology of Nuclear Energy, supported by Science and Technology Agency, Japan.

Authors wish to express their appreciation: to Dr. Koki SATO (NIRS), Chairman of Specialist Committee on Assessment and Reduction of Radiation Risks and to all members of the committee, for their kind support and useful suggestions; to Dr. Tetsuro OKANO of Kitasato University and to Mr. Shigehiro OZAWA of Sawa Kogyo Co., Ltd. for their contribution to the development of CHROMO MARKER; to Mr. Nobuo OKABE and to Mr. Ryo MIYAMOTO of Nikon Corporation, to Mr. Keiichi NISHIKAWA of Tokyo Dental College, to Dr. Nobuo FUKUDA of Toho University, to Dr. Norimasa NOHARA of NIRS, for their useful work and discussions; to NIRS staff, to Mr. Akira FURUKAWA for system improvements, to Ms. Fumi NAKAMOTO for designing the CHROMO ALGO software system, to Ms. Mieko KURANO and to Ms. Chie IITSUKA for programming on data base, to Ms. Tomoko KOKADO for improving the metaphase selection program, to Ms. Runa MOZUMDER for improving the English text, and to Ms. Masako NISHIKAWA for typing it.

\section{REFERENCES}

1. Lundsteen, C. and Piper, J. (1989) Automation of Cytogenetics, Springer-Veriag, BrusselsLuxembourg.

2. Lorch, T. (1990) Report, Interlaboratory exchange on automated chromosome aberration scoring. European Concerted Action on Automation in Cytogenetics, pp. 1-10, Neuherberg/Munich, 5-6 July.

3. Piper, J., Towers, S., Gordon, J., Ireland, J. and McDougall, D. (1988) Pattern recognition and artificial intelligence: in "Hypothesis combination and context sensitive classification for chromosome aberration scoring". Ed. Gelsema, E. S. and Kanal, L. N., pp.449-460, Elsevier Science Publishers B. V., North-Holland.

4. Lorch, T. Brochure of Meta Systems.

5. Yamamoto, M., Hayata, I., Nohara, N., Nakamoto, F., Kurano, M., Furuta, S., Okabe, N., Nishikawa, K., Fukuda, N. and Okano, T. (1991) Development of a high resolution chromosome image processing system for study purpose, NIRS-1000: CHROMO STUDY. Med. Imag. Tech. 9: 325-326.

6. Yamamoto, M., Hayata, I. and Onogi, K. (1989) Experimental comparison of spatial resolution between 500 and 1000 array imaging systems for microscopic specimens. Proc. XI European Workshop on Automated Cytogenetics, pp. 105, Besse en Chandesse, 14-17 September.

7. Yamamoto, M., Hayata, I., Nohara, N., Onogi, K., Miyamoto, R. and Okabe, N. (1990) Effect of 0.1 micro meter sampling with a high resolution television system for microscopy. Med. Imag. Tech. 8: 323-324.

8. Yamamoto, M. and Hayata, I. (1990) Development of high resolution image system for marking and scoring chromosome aberrations, NIRS-1000: CHROMO MARKER. Proc. XII European Workshop on Automated Cytogenetics, pp. 44-46, Segovia, 19-23 September.

9. Yamamoto, M. and Hayata, I. (1990) Development of high spatial resolution microscopic image system for marking and scoring chromosome aberrations. Med. Imag. Tech. 8: 451-457.

10. Kawashima, H., Imai, K., Fukuoka, H., Yamamoto, M. and Hayata, I. (1990) An algorithm for 
automatic detection of chromosome aberrations induced by radiation using features of gray level profile across the main axis of chromosome image. Med. Imag. Tech. 8: 458-475.

11. Inoue, S. (1989) Video Microscopy. Plenum Press, New York.

12. Hayata, I., Tabuchi, H., Furukawa, A., Okabe, N., Yamamoto, M. and Sato, K. (1991) Robot system for preparing lymphocyte chromosome. in this issue.

13. Yamada, K., Kakinuma, K., Tateya, H. and Toya, M. (1991) Development of an instrument for chromosome slide preparation. in this issue.

14. Finnon, P., Lloyd, D. C. and Edwards, A. A. (1986) An assessment of the metaphase finding capability of the Cytoscan 110. Mutation Research. 164: 101-108.

15. Okano, T., Yue, S., Yahata, K., Ohno, M., Funawatari, T., Hatakeyama, H., Shimomura, E., Ozawa, S. and Maeda, T. (1988) Application of image analyzer to chromosome analysis. Jpn. J. Clin. Pathol. 36(Suppl.): 349.

16. Mendelsohn, M. L., Mayall, B. H. and Prewitt, J. M. S. (1969) Approaches to the automation of chromosome analysis. in Image Processing in Biological Science, Ed. Ramsay D. M., Univ. Calfornia Press, Los Angeles, pp. 119-136.

17. Piper, J. and Granum, E. (1989) On fully automatic feature measurement for banded chromosome classification. Cytometry 10: 242-255. 